\title{
NONSTEROIDAL ANTI-INFLAMMATORY DRUGS (NSAIDS) IN COVID-19 PATIENT
}

\author{
Michal Paprockiఠ \\ Lazarski University, Faculty of Medicine, Warsaw, Poland
}

KEY WORDS: COVID-19, SARS-CoV-2, treatment; fever, nonsteroidal anti-inflammatory drugs, NSAIDs

Disaster Emerg Med J 2020; 5(2)

I read the article written by Smereka et al. [1] with great interest. The authors briefly and accessibly point out the problems of the current SARSCoV-2 coronavirus pandemic. One of the main symptoms indicated by patients with COVID-19 is high fever. Excessive fatigue and muscle pain are also indicated $[2,3]$. Therefore, one of the key questions arising in the context of COVID-19 is whether and, if so which non-steroidal anti-inflammatory drugs to use.

Opinions on the impact of non-steroidal anti-inflammatory drugs on the clinical course of COVID-19 caused by the new SARS-CoV-2 coronavirus are divided.

On 11 March 2020, "The Lancet Respiratory Medicine" published a publication indirectly addressing the impact of ibuprofen on the course of infection. The coronaviruses of acute respiratory syndrome SARS-CoV and SARS-CoV-2 bind to target cells by the angiotensin 2-converting enzyme (ACE 2), which is produced by epithelial cells of the lungs, intestines, kidneys and blood vessels. The expression of ACE 2 is significantly increased in type 1 or type 2 diabetic patients who are treated with ACE inhibitors and type II angiotensin receptor blockers (ARB). They also are used in the treatment of hypertension, which increases the secretion of ACE 2. ACE 2 can be increased by ibuprofen, among others. Increased expression of this enzyme facilitates COVID-19 infection. Therefore, the hypothesis has been raised that the treatment of diabetes and hypertension with ACE2 stimulant drugs increases the risk of development of severe and fatal COVID-19. ACE2 itself is a very important protein. Unfortunately, SARS-
CoV-2 coronavirus binds to it and uses it to enter the cells where it multiplies. $[4,5]$

According to the European Medicines Agency (EMA), there is currently no scientific evidence to confirm the link between NSAIDs and the deterioration of COVID-19. Healthcare personnel should consider all available treatment options, including paracetamol and NSAID-19, if a fever occurs in patients with COVID-19. It is recommended that these drugs are used in the lowest effective dose and for the shortest possible period. There is, therefore, no reason for patients taking ibuprofen to discontinue treatment. This is particularly important for patients receiving ibuprofen or NSAIDs due to chronic diseases [6].

On 19 March 2020, the World Health Organisation (WHO) issued an official statement in which it stated that the WHO is not against the use of ibuprofen based on existing data. However, it is aware of concerns about its use in the treatment of fever in people with COVID-19. It has been informed that physicians are being consulted and that it has not been informed of any negative effects beyond the usual side effects that limit the use of ibuprofen in some cases [7].

To summarize, there are no studies confirming higher mortality of COVID-19 when ibuprofen is used in the treatment. However, in doubtful cases, the use of paracetamol instead of ibuprofen is recommended but this refers only to its use without a physician's prescription [8].

\section{REFERENCES:}

1. Smereka J, Szarpak L, Filipiak KJ. Modern medicine in COVID-19 era. Disaster Emerg Med J. ; 2020, doi: 10.5603/DEMJ.a2020.0012. 
2. Lake MA. What we know so far: COVID-19 current clinical knowledge and research. Clin Med (Lond). 2020; 20(2): 124-127, doi: 10.7861/ clinmed.2019-coron, indexed in Pubmed: 32139372.

3. Ruetzler K, Szarpak L, Filipiak K, et al. The COVID-19 pandemic - a view of the current state of the problem. Disaster Emerg Med J. ; 2020, doi: 10.5603/DEMJ.a2020.0015.

4. Fang L, Karakiulakis G, Roth M. Are patients with hypertension and diabetes mellitus at increased risk for COVID-19 infection? Lancet Respir Med. 2020; 8(4): e21, doi: 10.1016/\$2213-2600(20)30116-8, indexed in Pubmed: 32171062.

5. Liu Z, Xiao X, Wei X, et al. Composition and divergence of coronavirus spike proteins and host ACE2 receptors predict potential intermediate hosts of SARS-CoV-2. J Med Virol. 2020 [Epub ahead of print], doi: 10.1002/jmv.25726, indexed in Pubmed: 32100877.
6. Day M. Covid-19: European drugs agency to review safety of ibuprofen. BMJ. 2020; 368: m1168, doi: 10.1136/bmj.m1168, indexed in Pubmed: 32205306.

7. Sodhi M, Etminan M. Safety of Ibuprofen in Patients with COVID-19; Causal or Confounded? Chest. 2020 [Epub ahead of print], doi: 10.1016/j.chest.2020.03.040, indexed in Pubmed: 32243944.

8. Gupta R, Misra A. Contentious issues and evolving concepts in the clinical presentation and management of patients with COVID-19 infectionwith reference to use of therapeutic and other drugs used in Co-morbid diseases (Hypertension, diabetes etc). Diabetes Metab Syndr. 2020 [Epub ahead of print]; 14(3): 251-254, doi: 10.1016/j. dsx.2020.03.012, indexed in Pubmed: 32247213. 